\title{
LA QUESTION DE LA PRODUGTION D'UN LAIT PRATIQUEMENT SAIN ET PUR.
}

\author{
par H.-R. BRÉDO, \\ Médecin Vétérinaire agréé du Gouvernement à Malines.
}

Dans tous les milieux on parle de lait sain et pur. On discute à l'infini les réglementations, etc., à faire appliquer pour obtenir théoriquement un lait idéalement sain et pur, et l'on semble oublier que comme tout idéal cette réalisation est impossible. Au lieu de s'attaquer directement au mal, on perd un temps précieux durant lequel des laitiers continuent à débiter un liquide frelaté, si pas toxique, à voler, empoisonner et même infecter leurs clients. On ne devrait pas perdre de vue que mieux vaut faire immediatement quelque chose d'imparfait que de tendre à réaliser limpossible.

L'inspection des viandes existe depuis des années et pourtant malgré les améliorations successives du șervice, elle est encore loin d'être parfaite. Quiconque en connait certains dessous doit reconnaitre qu'elle nécessite encore de rudes perfectionnements. Or, lorsqu'elle fut instituée, ceux qui se sont occupés de la question ne se sont fait aucune illusion. Ils savaient très bien que toute innovation est imparfaite et qu'il faut de très longues années avant de pouvoir espérer un service quelque peu efficace. L'expérience a démontré qu'on ne peut compter sur l'honnêteté commerciale des débitants ni espérer faire leur éducation immédiate. Le salut ne peut s'obtenir que par une surveillance serrée, une répression sévère et des pénalités sérieuses. La réglementation devrait considérer les débitants négiigents ou malhonnêtes, non comme des falsificateurs, des fraudeurs, mais commo des empoisonneurs ! Il serait donc indispensable que ceux-ci soient justiciables, non d'un tribunal de simple police, mais de la correctionnelle et même de la cour d'assises.

Mais, comment organiser pratiquement un tel service ? Avant tout il y aurait lieu de s'accorder sur les voies et moyens pour l'obtenir. Alors que les uns voudraient un service de contrôle libre dérivant de l'initiative privée, d'autres, au contruire, demandent l'intervention des autorités gouvernementales ou communales.

Nous estimons personnellement qu'un tel service doit être réglementé par le gouvernement et non par les communes et encore moins par des institutions privées qui lui font perdre toute son indépendance et lui enlèvent l'autorité dont il a un besoin absolu. De cette façon 
seule, il y aura moyen d'organiser une surveillance serrée, efficace et d'obtenir des sévères condamnations qui donneront à réfléchir à ceux qui seraient intentionnés de marcher dans la mauvaise voie.

En ce qui concerne les services à organiser, l'entente est loin d'exister. Alors que les uns exigent le contrôle de la production ayant pour base l'inspection sanitaire des vacheries, d'autres, reculant devant l'organisation d'un tel service, se contenteraient du contrôle du débit.

Et l'on s'entête à discuter vainement sans parvenir à s'entendre, on piétine sur place pendant que des laitiers continuent à commercer en affaiblissant, en intoxicant et en infectant nos enfants. nos affaiblis et nos malades. Tout cela provient de ce que l'on se maintient trop sur le terrain théorique alors que, vu l'urgence, on devrait agir rapidement et effectivement.

Dans la question du lait, nous pouvons distinguer 3 périodes :

$1^{\circ}$ La production et la récolte ;

$2^{\circ}$ Le transport ;

$3^{\circ}$ Le débit.

Nous ne nous étendrons pas sur les défectuosités actuelles, sur les inconvénients et les dangers en résultant, estimant que l'on a déjà discuté et écrit suffisamment sur ces matières pour pouvoir les considérer comme amplement étudiées.

Nous considérons ces 3 périodes comme liées indissolublement, mais ne pouvons admettre l'organisation d'un service d'inspection et de contrôle pour un seul des trois. En effet, en supposant que la production et la récolte soient parfaites, le transport ou le débit défectueux ou frauduleux fera perdre les bénéfices des qualités obtenues, et inversement.

Il faudra donc l'institution des 3 inspections et contrôles pour obtenir un produit relativement parfait et le maintenir tel. Les trois inspections sont solidaires les unes des autres, elles doivent éventuellement se contrôler mutuellement.

Nous avons pratiqué ces trois inspections et sommes parvenus à quelques améliorations. Nous sommes convaincu que de tels services arriveront à supprimer progressivementbeaucoup de défectuosités, etc.

L'inspection des ètables fut pratiquée dans la région de Malines. Gràce à nos conseils, nous sommes arrivé à faire améliorer l'état hygiénique, etc., de quelques étables en intervenant avant tout auprès des propriétaires. Nous avons donné des instructions pour remédier aux défectuosités de l'alimentation, pour l'écartement des mauvaises laitières, etc. et sommes parvenus à améliorer la récolte et les conditions de transport.

A Nimègue (Hollande), nous nous sommes occupé du service de 
contrôle hygiénique, bactériologique et chimique des laits débités en ville. Cet important service y a été organisé et est dirigé par notre estimé confrère $\mathrm{D}^{\mathrm{r}}$ Quadekker, Directeur de l'Abattoir de cette ville. Quoique loin d'être théoriquement parfait, il rend pratiquement de réels services.Les laits antihygiéniques,malpropres et autres . se font de plus en plus rares et les falsifications diminuent de jour en jour. Les analyses donnent non seulement des indications quant aux falsifications, mais encore quant laux conditions de la récolte, ete, elles constituent donc un véritable contrôle de la production.

Pour faire échec à l'organisation de l'inspection par le Gouvernement, certains font valoir que les finances de l'Etat ne permettent point les dépenses, qu’ils se plaisent à exagérer, nécessitées pour l'institution d'un tel service. A première vue, cet argument semble sérieux, quoique l'avenir de la race, le rétablissement des malades et l'alimentation générale méritent bien quelques sacrifices pécuniaires de la part du Gouvernement.

Seulement, on semble oublier que lorsque le service de l'inspection des viandes fut créé la situation était bien plus défavorable que l'actuelle. Alors, tout était à créer : abattoirs, laboratoires, etc., tandis que maintenant le nouveau service dispose déjà de toutes ces installations existantes.

Dès maintenant, on peut organiser un service en utilisant les établissements, institutions et personnel actuels. La pratique indiquera par après les perfectionnements indispensables à y porter progressivement.

On ne peut et ne doit pas exiger que tous les frais soient supportés par la caisse de l'Etat. Le service de l'inspection des viandes ne lui conte peu ou rien, il se suffit quasi à lui-même, il peut en être de même de ce nouveau service.

Nous ne demandons au Gouvernement qu'une bonne loi avec des sévères pénalités et de mettre à la disposition de ce service les établissements, le personnel discipliné dont il dispose. Le service vétèrinaire employé pour l'application des mesures de police sanitaire, les expertises des viandes, etc., pourra être utilisé pour l'inspeetion des étables, - la police assurera le respect des conditions imposées au transport et au débit; son service médical d'hygiène contrôlera l'état de santé du personnel humain, et les laboratoires d'analyses alimentaires détermineront, lors du débit, les laits antihygiéniques ou falsifiés. Les villes, communes, chargées de l'exécution de la loi agiront de même avec leurs services existants : abattoirs, laboratoires, etc. Ce faisant, nous sommes convaincu qu'en un temps très court on obtiendra une amélioration considérable des conditions de production et de débit du lait. 
A côté de la loi pénale et de la surveillance de la répression, les organismes existants : syndicats agricoles, syndicats d'élevage, associations de contrỏle de la production laitière, etc. interviendront pour instruire et éduquer les producteurs ainsi que les débitants. A cet - effet ils organiseront des conférences, des contrôles, des concours.

En leur allouant des subsides, les autorités pourront leur imposer certaines directives indispensables pour assurer une communauté de travail vers le but à atteindre.

On remarquera que, dans ce petit travail, nous avons intentionnellement évité le côté scientifique de la question. Comme nous. l'avons dit au début, nous estimons qu'elle est suffisamment étudiée pour permettre de s'engager dans la voie pratique. Nous sommes. persuadé que les divers organismes s'occupant de la défense des intérêts des consommateurs de lait, qui sont nombreux et puissants, instruits sur les dangers, etc. de la situation actuelle, pourraient exiger de nos mandataires une loi indispensable, profitant à la généralité. Cette législation s'obtiendra d'autant plus facilement. qu'elle n'exigera que peu ou point de sacrifices pécuniaires de la part. du Gouvernement. Nous insistons surtout sur ce point, c'est qu'il n'est pas nécessaire de nommer une légion de nouveaux fonctionnaires ni de créer de nombreux nouveaux établissements, nous sommes convaincu que le service pourra déjà fort bien débuter avec ce qui existe. Qu'on organise d'abord convenablement ne fut-ce qu'un service rudimentaire avec le matériel homme, etc. dont on dispose. Le mieux est souvent l'ennemi du bien et qui veut trop parfaire ne fait souvent rien. L'avenir décidera des améliorations devant et pouvant être portées; la question se résolvera mieux par la pratique que par toutes les commissions, ete., s'en tenant aux discussions théoriques.

\title{
L'AMELIORATION \\ DES GONDITIONS HYGIENIQUES DE LA \\ PRODUGTION DU LAIT DANS LE DEPARTEMENT DE SEINE-ET-OISE,
}

\author{
par M. ANDré-Max LEROY,
}

Chef de Travaux à l'Institut National Agronomique.

Secrétaire technique du Comité de l'Elevage de Seine-et-Oise.

En raison de sa proximité de l'agglomération parisienne, le dépar. tement de Seine-et-Oise possède de nombreuses vacheries, qui fournissent du lait destiné dans bien des cas à la consommation en nature, 